\title{
Comportamentos suicidários: caracterização e discussão de fatores de vulnerabilidade
}

Andreia Filipa Araújo da Silva Ramôa, ${ }^{1}$ Célia Soares, ${ }^{2}$ Joana Castanheira, ${ }^{3}$ José Sequeira, ${ }^{4}$ Natália Fernandes, ${ }^{2}$ Sónia Azenha ${ }^{2}$

\begin{abstract}
RESUMO
Objetivos: Caraterizar a população de doentes observados em primeira consulta na Unidade de Comportamentos Suicidários (UCS) do serviço de psiquiatria do Hospital de Braga (HB) durante os anos 2014-2015 e identificar características associadas a maior risco de comportamentos suicidários de repetição e uso de métodos violentos.

Tipo de estudo: Estudo observacional, transversal, descritivo e analítico.

Local: Hospital de Braga.

População: Doentes que tiveram primeira consulta na UCS do serviço de psiquiatria do HB no período compreendido entre 01/01/2014 e 31/12/2015.

Métodos: Recolha de dados da rotina da consulta. Recolha de variáveis sociodemográficas, psicossociais, circunstanciais do comportamento suicidário e clínicas. Os dados foram colhidos através do processo clínico informático e analisados no programa SPSS ${ }^{\circledR}$, v. 21 . Foi realizada uma análise descritiva univariada e bivariada. Seguidamente foi criado um modelo de regressão logística binária para identificar possíveis fatores de risco associados aos comportamentos repetitivos e uso de métodos mais violentos.

Resultados: Foram incluídos no estudo 154 indivíduos, maioritariamente mulheres (70,8\%), com média de idades de 39,7 anos. A maioria era casada (51,9\%). Os meses com períodos diurnos mais curtos foram aqueles em que se observaram maior número de tentativas de suicídio. A ingestão medicamentosa foi o método mais frequente (68,2\%). Os métodos mais violentos e letais foram mais usados por homens $(p=0,036)$. O consumo de substâncias foi associado ao uso de métodos mais violentos $(p=0,045)$. A existência de perturbação da personalidade $(p=0,001)$ e a terapêutica com antidepressivos $(p=0,028)$ foram fatores de risco para comportamentos suicidários de repetição. Este estudo revelou ainda que a maioria (72\%) das pessoas recorreu ao seu médico de família nos 12 meses prévios à tentativa de suicídio.

Conclusões: Neste estudo, o diagnóstico mais frequente foi a depressão. Este estudo permitiu identificar, por um lado, a perturbação da personalidade e o uso de antidepressivos como fatores de risco associados ao comportamento suicidário repetitivo. Por outro lado, ajudou a definir o género masculino e o consumo de substâncias como fatores de risco para o uso de métodos mais violentos e letais na tentativa de suicídio. Este conhecimento é essencial para a prevenção da mortalidade associada aos comportamentos suicidários, nomeadamente entre médicos de família, que se encontram numa posição privilegiada para conhecimento do indivíduo e seu contexto. São, no entanto, necessários mais estudos para reforçar a influência dos fatores considerados de risco na população, uma vez que vários destes carecem ainda de evidência epidemiológica consistente.
\end{abstract}

Palavras-chave: Suicídio; Estudo sobre vulnerabilidade.

\section{INTRODUÇÃO}

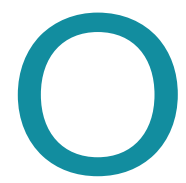

suicídio é um importante problema de saúde pública que, pela sua complexidade e natureza intrigante, obriga à aquisição de competências de avaliação de risco e adoção de estratégias preventivas eficazes. ${ }^{1}$

O suicídio é definido pela Organização Mundial da

1. USF Gualtar

2. Hospital de Braga

3. USF St ${ }^{\circ}$ António

4. USF + Carandá
Saúde (OMS) como um ato deliberado, iniciado e levado a cabo por um indivíduo com pleno conhecimento ou expectativa de um resultado fatal. ${ }^{2}$ Os comportamentos suicidários constituem qualquer ato através do qual um individuo causa uma lesão em si mesmo, independentemente do grau de intenção letal. Por sua vez, nos comportamentos autolesivos (termo que substitui o termo clássico parassuicídio) não existe intencionalidade suicida, incluindo-se nestes os atos autolesivos intencionais. Os comportamentos autolesivos são considerados o principal preditor para o suicídio. ${ }^{2}$ 
De acordo com os dados da OMS, cerca de 800.000 pessoas morrem por suicídio a cada ano, estimando-se que um número bem maior faça tentativas de suicídio. Em 2012, o suicídio foi a segunda causa de morte na população com idades compreendidas entre os 15-29 anos. Estima-se que, em 2020, o número de suicídios atinga os 1,5 milhões. ${ }^{3}$ Em Portugal, segundo dados de 2011 do Instituto Nacional de Estatística, o fenómeno atinge cerca de 9.6/100.000 habitantes. ${ }^{4}$

O conhecimento e a identificação dos fatores de risco para o suicídio por parte dos profissionais de saúde permitem uma avaliação e intervenção mais eficazes no que se refere à prevenção de comportamentos suicidários. Existem várias categorias de fatores de risco identificadas. Entre os fatores de risco sociodemográficos, a maior parte dos estudos disponíveis mostra que o sexo masculino apresenta três vezes maior risco de suicídio e que as taxas de suicídio são mais altas nos idosos. No entanto, um estudo multicêntrico europeu, desenvolvido pela OMS, encontrou taxas de tentativas de suicídio superiores no género feminino e em idades mais jovens. $\mathrm{O}$ desemprego, o isolamento social, o divórcio e os baixos níveis de educação e socioeconómicos estão também associados a maior risco de comportamentos suicidários. ${ }^{5}$

A sazonalidade também tem vindo a demonstrar influência no suicídio e tentativas de suicídio, sendo descrito na literatura um pico na primavera e no verão. ${ }^{6-7} \mathrm{Re}-$ lativamente ao método utilizado, os estudos disponíveis são consensuais, descrevendo o género masculino como utilizador de métodos mais violentos..$^{8-12}$

A história familiar e pessoal de patologia psiquiátrica ou comportamentos suicidários, comorbilidades médicas e acontecimentos de vida adversos também têm sido associados a um maior risco de suicídio. ${ }^{2}$ Relativamente aos diagnósticos psiquiátricos, a depressão, o consumo de substâncias de abuso, a esquizofrenia e as perturbações da personalidade têm revelado maior associação com comportamentos suicidários, constituindo fatores de risco de suicídio. ${ }^{13}$

Os médicos de família desempenham um papel fundamental na prevenção dos comportamentos suicidários, sendo essencial o conhecimento das características da população que tenta o suicídio. Efetivamente são eles que acompanham o doente a longo prazo, estando numa posição privilegiada para detetar fatores de risco preditores destes comportamentos. ${ }^{14}$
Dada a dimensão deste problema, é imprescindível uma melhor compreensão do perfil do indivíduo com comportamentos suicidários. A existência de uma consulta especializada para acompanhamento destes doentes constitui uma oportunidade de análise da mesma, permitindo averiguar se existe uma concordância dos fatores de vulnerabilidade para comportamentos suicidários com os descritos na literatura.

Este trabalho de investigação tem como objetivo caracterizar a população de doentes acompanhados em consulta na Unidade de Comportamentos Suicidários (UCS) do serviço de psiquiatria do Hospital de Braga (HB), que tiveram primeira consulta durante os anos 2014-2015. Como objetivos específicos, os autores pretenderam estabelecer associação entre fatores sociodemográficos, sazonalidade, história pessoal e familiar de patologia psiquiátrica e comportamentos suicidários. Foi ainda objetivo identificar as características associadas a maior risco de comportamentos suicidários de repetição e uso de métodos violentos.

\section{MÉTODOS}

Realizou-se um estudo observacional, transversal, descritivo e analítico que decorreu durante o mês de março de 2016 no serviço de psiquiatria do HB.

A população em estudo foi constituída por doentes adultos, com idade $\geq 18$ anos, que tiveram primeira consulta na UCS do serviço de psiquiatria do HB no período compreendido entre 01-01-2014 e 31-12-2015. Foram excluídos os doentes que não apresentaram comportamentos suicidários e cuja referenciação para a consulta se deveu à presença de fatores de risco para o comportamento suicidário, nomeadamente a presença de ideação suicida. A população definida foi estudada na sua totalidade, não tendo sido selecionada nenhuma amostra. $\mathrm{O}$ período de estudo foi definido tendo por base questões logísticas, nomeadamente a existência de uma percentagem significativa de registos em papel anteriores a 2014, que poderiam comprometer a colheita dos dados. Esta foi realizada por quatro dos investigadores do estudo.

Para a recolha dos dados foram consultados os processos clínicos informáticos - utilizando os programas Gllint ${ }^{\circledR}$ e PDS $®$. Foram considerados os dados relativos não só à primeira consulta compreendida no período em estudo, assim como todas as consultas subsequentes, de modo a minimizar vieses derivados da ausência ou incorreção de registos. De modo a garantir a total confi- 
dencialidade e anonimato dos resultados foi atribuído a cada utente da população estudada um código identificativo. O único ficheiro com a ligação entre o código identificativo e a identificação do utente ficou protegido por palavra-passe, com acesso exclusivo dos investigadores.

As variáveis estudadas foram as seguintes: idade, género, escolaridade, estado civil, situação habitacional (sozinho, em casa própria), situação profissional, localidade de residência, data da tentativa de suicídio, motivo da tentativa de suicídio, método utilizado, tentativas de suicídio prévias, pedido de ajuda, diagnóstico psiquiátrico, presença de perturbação de personalidade, seguimento prévio em consulta de psiquiatria, antecedentes familiares de suicídio, história de abuso de substâncias e presença de comorbilidades médicas. A descrição pormenorizada das variáveis encontra-se no Quadro I.

O protocolo do estudo foi submetido à Comissão de Ética para a Saúde do HB (CESHB), que emitiu parecer favorável para a realização do mesmo.

Os dados foram analisados no programa SPSS $®$ v. 21, utilizando um nível de significância estatística de $5 \%(p<0,05)$, tendo sido realizada uma análise descritiva univariada ebivariada. Para análise bivariada foram utilizados os testes Qui-quadrado e T-student para as variáveis qualitativas e quantitativas, respetivamente. Posteriormente foi feita uma análise multivariada, através da criação de um modelo de regressão logística binária, para identificar possíveis fatores de risco associados aos comportamentos repetitivos e uso de métodos mais violentos. Esta análise multivariada teve como principal vantagem, em relação à análise bivariada, a neutralização das variáveis de confundimento que pudessem não estar identificadas pelos investigadores.

Os investigadores identificam como principal viés do estudo o viés de informação, pela extração dos dados ter sido feita retrospetivamente.

Na inexistência de dados no processo clínico em relação a alguma variável do estudo, esta foi codificada na base de dados como missing. Desta forma, a análise estatística não ficou comprometida porque os testes foram aplicados apenas para os indivíduos com essa informação, tendo sido sempre considerado o tamanho do grupo estudado (n) para garantir a aplicabilidade dos testes.

\section{RESULTADOS}

Durante o período compreendido entre 01-01-2014 e 31-12-2015 foram realizadas 186 primeiras consultas na
UCS do serviço de psiquiatria do HB. Os dados foram colhidos retrospetivamente através da análise dos processos clínicos informáticos. Foram excluídos 32 indivíduos por não terem realizado nenhuma tentativa de suicídio. Assim, foram incluídas neste estudo 154 pessoas.

\section{Fatores sociodemográficos e sazonalidade}

Foram estudadas 109 pessoas do género feminino (70,8\%) e 45 pessoas do género masculino, com idade mediana de 39 anos e idade média de 39,7 anos. A idade média foi de 39,5 anos para o género feminino e de 40,2 anos para o género masculino, sem diferença estatisticamente significativa na média de idade para o género $(t=0,285$; $p=0,776$ ). A variável «escolaridade» foi obtida em apenas $39 \%$ dos casos. Destes, $23,4 \%$ tinha o $4^{\circ}$ ano de escolaridade, ou menos, e 13,3\% tinha o ensino superior. A maioria dos indivíduos era casada $(51,9 \%)$ e apenas $7,8 \%$ vivia sozinho. No que respeita à situação profissional, 51,4\% estava ativo e cerca de $32 \%$ desempregado. A maioria das pessoas observadas em primeira consulta na UCS do serviço de psiquiatria no $\mathrm{HB}$ pertencia aos conselhos de Braga e Barcelos. A taxa de pessoas observadas nesta consulta é também maior nestes concelhos - de 42,4/100.000 habitantes em Braga e de 35,7/100.000 habitantes em Barcelos -, sendo a taxa média de pessoas observadas nesta consulta de 23,6/100.000 habitantes (Quadros II e III).

O número de tentativas de suicídio nos anos de 2014 e 2015 foi semelhante (68 e 66, respetivamente). Os meses com maior número de tentativas de suicídios foram janeiro, fevereiro, agosto, novembro e dezembro (Figura 1).

\section{Motivo e método da tentativa de suicídio}

Os conflitos conjugais ou no relacionamento afetivo foram o principal motivo da tentativa de suicídio, responsável por cerca de $42 \%$ dos casos. Outros conflitos familiares motivaram $16,2 \%$ das tentativas de suicídio observadas e o agravamento da sintomatologia depressiva e/ou ansiosa foi responsável por 8,8\% das tentativas registadas.

Tentou perceber-se se havia um número maior de tentativas de suicídio por agravamento da sintomatologia depressiva e/ou ansiosa nos meses de inverno; no entanto, não se verificou esta relação $(x=0,804 ; p=0,446)$. Da mesma forma, não se encontrou relação entre os conflitos conjugais e as tentativas de suicídio realizadas no mês de agosto $(x=0,133 ; p=0,786)$ ou nos meses de inverno $(x=0,210 ; p=0,735)$. 


\begin{tabular}{|c|c|c|c|}
\hline Variável & Descrição & Categoria & Código \\
\hline Idade & Idade, em anos, à data da primeira consulta & $18,19,20 \ldots$ & - \\
\hline Sexo & Género do indivíduo estudado & $\begin{array}{l}\text { Masculino } \\
\text { Feminino }\end{array}$ & $\begin{array}{l}1 \\
2\end{array}$ \\
\hline Escolaridade & Habilitação literária do indivíduo estudado & $\begin{array}{c}<4 \\
4^{\circ} \\
6^{\circ} \\
9^{\circ} \\
12^{\circ} \\
\text { Bacharelato } \\
\text { Licenciatura } \\
\text { Mestrado } \\
\text { Doutoramento }\end{array}$ & $\begin{array}{l}0 \\
1 \\
2 \\
3 \\
4 \\
5 \\
6 \\
7 \\
8\end{array}$ \\
\hline Estado civil & Estado civil da pessoa & $\begin{array}{l}\text { Solteiro } \\
\text { Casado } \\
\text { Divorciado } \\
\text { Viúvo }\end{array}$ & $\begin{array}{l}0 \\
1 \\
2 \\
3\end{array}$ \\
\hline Vive sozinho & & $\begin{array}{l}\text { Não } \\
\text { Sim }\end{array}$ & $\begin{array}{l}0 \\
1\end{array}$ \\
\hline Situação profissional & & $\begin{array}{c}\text { Ativo } \\
\text { Desempregado } \\
\text { Reformado } \\
\text { Desconhecido }\end{array}$ & $\begin{array}{l}1 \\
2 \\
3 \\
4\end{array}$ \\
\hline Profissão & Atividade profissional que exerce & NA & NA \\
\hline Localidade & Concelho onde habita & NA & NA \\
\hline Data da tentativa de suicídio & Mês e ano da primeira tentativa de suicídio & NA & NA \\
\hline Motivo & Descrição do motivo identificado pelo utente & NA & NA \\
\hline Método & Método pelo qual tentou o suicídio & $\begin{array}{l}1 \\
2 \\
3 \\
4 \\
5 \\
6 \\
7 \\
8\end{array}$ & $\begin{array}{c}\text { IMV } \\
\text { Tóxicos } \\
\text { Arma de fogo } \\
\text { Enforcamento } \\
\text { Flebotomia } \\
\text { Afogamento } \\
\text { Precipitação } \\
\text { Trucidação }\end{array}$ \\
\hline Método violento & $\begin{array}{l}\text { Corresponde aos métodos: arma de fogo, } \\
\text { enforcamento, precipitação e trucidação }\end{array}$ & $\begin{array}{l}0 \\
1\end{array}$ & $\begin{array}{l}\text { Não } \\
\text { Sim }\end{array}$ \\
\hline Repetidor & Fez outras tentativas de suicídio? & $\begin{array}{l}0 \\
1\end{array}$ & $\begin{array}{l}\text { Não } \\
\text { Sim }\end{array}$ \\
\hline Ajuda & Pediu ajuda? & $\begin{array}{l}0 \\
1\end{array}$ & $\begin{array}{l}\text { Não } \\
\text { Sim }\end{array}$ \\
\hline
\end{tabular}




\begin{tabular}{|c|c|c|c|}
\hline Variável & Descrição & Categoria & Código \\
\hline Diagnóstico & $\begin{array}{l}\text { Diagnóstico principal registado no processo } \\
\text { clínico }\end{array}$ & $\begin{array}{l}1 \\
2 \\
3 \\
4 \\
5 \\
6 \\
7 \\
8 \\
9\end{array}$ & $\begin{array}{l}\text { Depressão } \\
\text { PP A } \\
\text { PP B } \\
\text { PP C } \\
\text { P. bipolar } \\
\text { P. ansiedade } \\
\text { Psicose } \\
\text { Alcoolismo } \\
\text { Outros }\end{array}$ \\
\hline Seguimento em psiquiatria & $\begin{array}{l}\text { Utente já se encontrava a ser seguido na } \\
\text { consulta de psiquiatria anteriormente à } \\
\text { tentativa de suicídio? }\end{array}$ & $\begin{array}{l}0 \\
1\end{array}$ & $\begin{array}{l}\text { Não } \\
\text { Sim }\end{array}$ \\
\hline Tratamento psiquiátrico prévio & $\begin{array}{l}\text { Classe de psicofármacos que o utente se } \\
\text { encontrava a fazer aquando da tentativa de } \\
\text { suicídio }\end{array}$ & NA & NA \\
\hline Antecedentes familiares de suicídio & $\begin{array}{l}\text { Existência conhecida de antecedentes de } \\
\text { suicídio nos familiares }\end{array}$ & $\begin{array}{l}0 \\
1\end{array}$ & $\begin{array}{l}\text { Não } \\
\text { Sim }\end{array}$ \\
\hline Abuso de substâncias & Consumo de substâncias de abuso & $\begin{array}{l}0 \\
1 \\
2 \\
3 \\
4 \\
5\end{array}$ & $\begin{array}{l}\text { Não } \\
\text { Álcool } \\
\text { Canabinóides } \\
\text { Cocaína } \\
\text { Heroína } \\
\text { Outros }\end{array}$ \\
\hline Comorbilidades & Descrever comorbilidades médicas associadas & NA & NA \\
\hline CSP & $\begin{array}{l}\text { Consulta nos CSP nos } 12 \text { meses prévio à } \\
\text { tentativa de suicídio }\end{array}$ & $\begin{array}{l}0 \\
1\end{array}$ & $\begin{array}{l}\text { Não } \\
\text { Sim }\end{array}$ \\
\hline
\end{tabular}

Legenda: NA - Não aplicável; IMV - Ingestão medicamentosa voluntária; CSP - Cuidados de saúde primários.

A ingestão medicamentosa foi o método mais frequentemente observado - em $70 \%$ dos casos. Os métodos violentos, que incluem a utilização de arma de fogo, o enforcamento, a precipitação e a trucidação por comboio, foram utilizados em $8,7 \%$ dos casos. A maioria dos indivíduos que tentaram o suicídio pediu ajuda $(67,2 \%)$ antes ou depois da tentativa. O comportamento repetidor verificou-se em $32,7 \%$ dos indivíduos. No Quadro IV encontram-se as principais características da tentativa de suicídio.

\section{Caracterização psiquiátrica}

A depressão foi mais frequentemente definida como diagnóstico principal, estabelecida em $51,3 \%$ dos casos. A existência de perturbação da personalidade foi o principal diagnóstico em 16,2\% e a perturbação de adaptação em $14,9 \%$. Cerca de $30 \%$ das pessoas incluídas no estudo tinham o diagnóstico (primário ou secundário) de perturbação da personalidade. Destas, $76,1 \%$ tinha perturbação da personalidade do cluster $\mathrm{B}$, onde se incluem as perturbações de personalidade borderline. A maioria das pessoas estudadas $(56,5 \%)$ não tinha seguimento prévio na consulta de psiquiatria e cerca de $72 \%$ recorreu aos cuidados de saúde primários nos 12 meses prévios à tentativa de suicídio. $\mathrm{O}$ abuso de substâncias estava presente em 17,3\% dos casos, sendo o álcool a principal substância consumida. O abuso de substâncias foi mais frequente no género masculino 


\begin{tabular}{|c|c|}
\hline \multicolumn{2}{|c|}{$\begin{array}{l}\text { QUADRO II. Características sociodemográficas da } \\
\text { população estudada }\end{array}$} \\
\hline Variável & $n(\%)$ \\
\hline \multicolumn{2}{|l|}{ Género $(n=154)$} \\
\hline Masculino & $45(29,2)$ \\
\hline Feminino & $109(70,8)$ \\
\hline \multicolumn{2}{|c|}{ Escolaridade $(n=60)$} \\
\hline$\leq 4^{\circ}$ ano & $14(21,7)$ \\
\hline $6^{\circ}$ ano & $8(13,3)$ \\
\hline $9^{\circ}$ ano & $16(26,7)$ \\
\hline $12^{\circ}$ ano & $14(23,3)$ \\
\hline$>12^{\circ}$ ano & $8(13,3)$ \\
\hline \multicolumn{2}{|c|}{ Estado civil $(n=154)$} \\
\hline Solteiro & $49(31,8)$ \\
\hline Casado & $80(51,9)$ \\
\hline Divorciado & $20(13)$ \\
\hline Viúvo & $5(3,2)$ \\
\hline \multicolumn{2}{|c|}{ Vive sozinho $(n=154)$} \\
\hline Sim & $12(7,8)$ \\
\hline Não & $142(92,2)$ \\
\hline \multicolumn{2}{|c|}{ Situação profissional $(n=144)$} \\
\hline Ativo & $74(51,4)$ \\
\hline Desempregado & $46(31,9)$ \\
\hline Reformado & $15(10,4)$ \\
\hline Estudante & $9(6,3)$ \\
\hline Idade & Média (Desvio-padrão) \\
\hline Total & $39,7( \pm 15)$ \\
\hline Masculino & $40,2( \pm 18,6)$ \\
\hline Feminino & $39,5( \pm 13,2)$ \\
\hline
\end{tabular}

$\begin{aligned} & \text { QUADRO III. Distribuição da população e taxa de tentativa de suicídio por concelho de } \\
& \text { residência }\end{aligned}$
\begin{tabular}{l|r|r|r} 
Concelho $(n=154)$ & $n(\%)$ & População residente (INE) & $\begin{array}{c}\text { Taxa de tentativas de suicídio } \\
\text { (por 100.000 habitantes) }\end{array}$ \\
\hline Braga & $77(50)$ & 181.494 & 42,4 \\
\hline Barcelos & $43(27,9)$ & 120.391 & 35,7 \\
\hline Vila Verde & $9(5,8)$ & 47.888 & 18,8 \\
\hline Esposende & $6(3,9)$ & 34.254 & 17,5 \\
\hline Póvoa de Lanhoso & $5(3,2)$ & 21.886 & 22,8 \\
\hline Outros & $14(9,1)$ & & \\
\hline
\end{tabular}

( $x=21,613 ; p=0,001$ ). Das pessoas estudadas, $38,5 \%$ estava medicada com antidepressivos. As comorbilidades médicas estiveram presentes em $45,5 \%$ dos casos. A maioria (72\%) das pessoas do estudo recorreu ao seu médico de família nos 12 meses prévios à tentativa de suicídio (Quadro V).

Fatores associados à utilização de métodos violentos e aos comportamentos suicidários de repetição

Foi construído um modelo de regressão logística binário para verificar se existiam fatores de risco associados à utilização de métodos violentos e comportamentos repetidos. Para isso foram estudadas as variáveis "género», «viver sozinho», "perturbação da personalidade», «uso de antidepressivos» $\mathrm{e}$ «abuso de substâncias».

Verificou-se que o género masculino $(B=1,894 ; O R=2,939$; IC=[1,453-4,328]; $p=0,036)$ e o consumo de substâncias de abuso ( $\mathrm{B}=1,076$; $\mathrm{OR}=2,654$; $\mathrm{IC}=[1,189-3,678] ; p=0,045$ ) estão associados ao uso de métodos mais violentos.

A existência de perturbação da personalidade $(\mathrm{B}=1,479 ; \mathrm{OR}=4,390 ; \mathrm{IC}=[1,854-10,396] ; p=0,001)$ e a terapêutica com antidepressivos $(\mathrm{B}=0,856$; $\mathrm{OR}=2,354$; $\mathrm{IC}=[1,003-5,552] ; p=0,049)$ foram fatores associados aos comportamentos suicidários de repetição (Quadro VI).

\section{DISCUSSÃO}

Os estudos epidemiológicos referentes aos comportamentos suicidários não letais têm-se baseado em metodologias denominadas por «áreas de captação», que correspondem a áreas geográficas de dimensão geralmente mais restrita do que a de um país, para as quais se procede a um processo de monitorização tendo em vista o registo de todos os casos que ocorram num determinado período de tempo. ${ }^{2}$ Não existem, até ao momento, estudos epidemiológicos que permitam uma compreensão completa e global do fenómeno, sendo os conhecimentos atuais provenientes sobretudo da generalização de resultados obtidos através de amostras populacionais selecionadas por conveniência.

Nesses moldes, globalmente este estudo observacional permitiu inferir acer- 


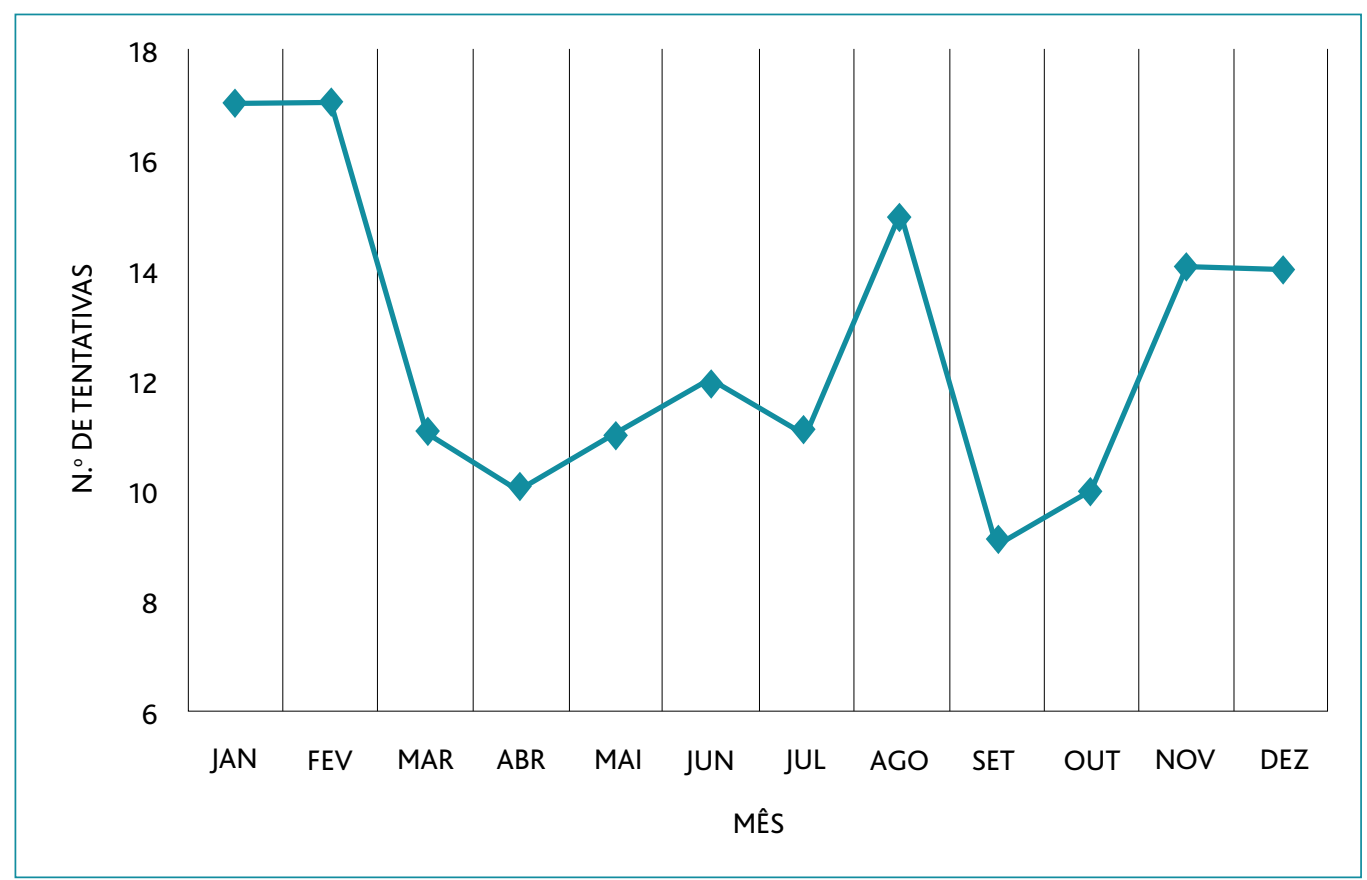

Figura 1. Distribuição das tentativas de suicídio por mês.

ca das características associadas a maior risco de comportamentos de repetição, nomeadamente o diagnóstico de perturbação da personalidade e o uso de antidepressivos. Adicionalmente permitiu associar o género masculino e o abuso de substâncias ao uso de métodos mais violentos. Seguidamente abordam-se, em particular, aspetos relevantes em relação aos resultados apresentados.

\section{Fatores sociodemográficos}

No que se refere aos fatores sociodemográficos verificou-se neste estudo uma relação feminino/masculino de aproximadamente dois para um, mais elevada do que aquelas que foram reportadas num estudo multicêntrico europeu, realizado pela OMS, envolvendo 15 áreas de captação noutros países, mas semelhante à relação observada num estudo sobre epidemiologia destes comportamentos no concelho de Coimbra, incidindo na década de $90.5,15$

Contrariamente a outros estudos, não se verificou um predomínio em idades jovens nem uma diferença significativa no que refere à idade para o género. ${ }^{15-17}$

A maioria da amostra pertenceu ao estado civil «casado» e estava numa situação profissional ativa, ao invés do que tem sido reportado noutros estudos, que referem o di- vórcio e o desemprego como fatores de risco para os comportamentos suicidários. ${ }^{5}$ Esta diferença de resultados poderá ilustrar um maior contributo de outras variáveis nesta amostra ou ser decorrente das limitações do estudo.

\section{Sazonalidade}

É referida, na literatura, uma tendência sazonal para o suicídio consumado e para as tentativas de suicídio, com um pico na primavera e no verão, o que sugere a presença de um substrato biológico na etiopatogénese destes comportamentos. ${ }^{6-7}$ Lester, em 1985, descreveu uma tendência bimodal, com picos no outono e no inverno, bem como ausência de sazonalidade para uso de métodos não violentos, que predominam nesta amostra, embora estudos posteriores não tenham corroborado esta hipótese de forma consistente. ${ }^{18}$

Neste estudo verificou-se uma maior tendência de comportamentos suicidários nos meses de inverno e um pico no mês de agosto. Atendendo a que o agravamento depressivo foi um dos principais diagnósticos psiquiátricos na origem da tentativa de suicídio, e sabendo-se que existe uma tendência para um agravamento psicopatológico da depressão nos meses com período diurno mais reduzido, esta poderia ser uma explicação possível. ${ }^{7}$ Por outro 
lado, os conflitos conjugais estiveram presentes num número significativo de casos, como precipitantes da tentativa de suicídio. Uma explicação possível para o pico observado no verão poderia ser o período de férias e a maior disponibilidade para a família, com consequente aumento dos conflitos conjugais. No entanto, nenhuma destas hipóteses se verificou neste estudo.

\section{Caracterização psiquiátrica}

As perturbações do humor (particularmente a depressão), o consumo de substâncias de abuso, a esquizofrenia e as perturbações da personalidade são conhecidos como os principais diagnósticos psiquiátricos associados aos comportamentos suicidários. ${ }^{13}$ Neste estudo, a depressão foi o principal diagnóstico estabelecido, seguido pelas perturbações da personalidade. Outros diagnósticos, como a esquizofrenia e o consumo de substâncias de abuso, nomeadamente o alcoolismo, não foram diagnósticos frequentes neste estudo, o que poderá ser explicado pelo facto de existirem, no serviço de psiquiatria do $\mathrm{HB}$, consultas próprias para estas perturbações, não tendo sido contabilizadas as tentativas de suicídio nestas consultas.

Vários fatores (sociais, culturais, de disponibilidade) contribuem para a escolha do método para a tentativa de suicídio, o que leva a que os métodos mais frequentemente utilizados variem de acordo com a região geográfica. ${ }^{8,19} \mathrm{O}$ suicídio por arma de fogo é o método mais comum nos Estados Unidos da América; as precipitações têm maior destaque em sociedades predominantemente urbanas, como Hong Kong, Luxemburgo e Malta; nos países latino-americanos, como Portugal, as intoxicações são o principal problema. ${ }^{8}$ Este estudo vai de encontro ao descrito, sendo as ingestões medicamentosas e a ingestão de outros tóxicos os métodos mais frequentes, escolhidos em $81 \%$ dos casos. A combinação de métodos tem sido descrita como sendo mais comum no género masculino. ${ }^{20-21}$ Neste estudo apenas dois indivíduos utilizaram mais do que um método, sendo ambos do género masculino. No entanto, o número limitado de casos não permite estabelecer conclusões.

No que respeita aos pedidos de ajuda, neste estudo não se verificou diferença estatisticamente significativa entre os géneros, ao contrário do que se verifica noutros estudos, em que a ajuda é mais frequentemente pedida pelo género feminino. ${ }^{9,22-23}$

\section{QUADRO IV. Caracterização da tentativa de suicídio}

\begin{tabular}{l|c} 
Variável & $n(\%)$ \\
\hline Motivo ( $\boldsymbol{n}=148)$ & $62(41,9)$ \\
Conflitos conjugais /no relacionamento afetivo & $24(16,2)$ \\
Outros conflitos familiares & $13(8,8)$ \\
Agravamento da sintomatologia depressiva/ansiosa & $8(5,4)$ \\
Conflitos laborais & $7(4,7)$ \\
Sentimentos de desesperança & $34(22,1)$ \\
Outro & \\
\hline Método (n=150) & $105(70)$ \\
Ingestão medicamentosa & $17(11,3)$ \\
Ingestão de tóxicos & $12(8)$ \\
Flebotomia & $7(4,7)$ \\
Precipitação & $4(2,7)$ \\
Enforcamento & $1(0,7)$ \\
Arma de fogo & $1(0,7)$ \\
Afogamento & $1(0,7)$ \\
Trucidação por comboio & $1(0,7)$ \\
Flebotomia + Ingestão medicamentosa & $1(0,7)$ \\
Flebotomia + Ingestão de tóxicos & \\
\hline Comportamento repetidor? $(\boldsymbol{n}=153)$ & $50(32,7)$ \\
Sim & $103(67,3)$ \\
\hline Não & $25(21,5)$ \\
\hline Pedido de ajuda? ( $=116)$ & $53(45,7)$ \\
Sim & $38(32,8)$ \\
- Sim, antes & \\
- Sim, depois & \\
Não & \\
& \\
\hline
\end{tabular}

Neste estudo, como descrito em alguns estudos, o consumo de álcool e drogas é mais comum no género masculino $(p=0,001){ }^{24}$

\section{Fatores associados ao uso de métodos violentos}

Na Europa, o género masculino tem uma maior taxa de suicídios concretizados, o que indica diferenças na letalidade dos comportamentos suicidários relativamente ao género. Efetivamente é unânime na literatura que o género masculino é conhecido por tentar o suicídio utilizando métodos mais violentos..$^{8-12}$ Neste estudo, os resultados foram concordantes com os descritos, verificando-se uma associação significativa entre a utilização de métodos violentos e o género masculino. Apesar de ser mais frequente no gé- 
QUADRO V. Características clínicas da população estudada

\begin{tabular}{|c|c|}
\hline Variável & $n(\%)$ \\
\hline \multicolumn{2}{|l|}{ Diagnóstico principal $(n=154)$} \\
\hline Depressão & $79(51,3)$ \\
\hline Perturbação da personalidade & $25(16,2)$ \\
\hline Perturbação de adaptação & $23(14,9)$ \\
\hline Perturbação de ansiedade & $8(5,2)$ \\
\hline Alcoolismo & $4(2,6)$ \\
\hline Perturbação bipolar & $2(1,3)$ \\
\hline Outros & $13(8,4)$ \\
\hline \multicolumn{2}{|l|}{ Perturbação da personalidade? ( $n=153)$} \\
\hline Sim & $46(30,1)$ \\
\hline Não & $107(69,9)$ \\
\hline \multicolumn{2}{|l|}{ Seguimento prévio em psiquiatria? $(n=154)$} \\
\hline Sim & $67(43,5)$ \\
\hline Não & $87(56,5)$ \\
\hline \multicolumn{2}{|l|}{ Consumo de substâncias de abuso? $(n=139)$} \\
\hline Sim & $24(17,3)$ \\
\hline - Álcool & $18(12,9)$ \\
\hline - Canabinóides & $2(1,4)$ \\
\hline - Outros & $4(2,9)$ \\
\hline Não & $115(82,7)$ \\
\hline \multicolumn{2}{|l|}{ Uso de antidepressivos? $(n=148)$} \\
\hline Sim & $57(38,5)$ \\
\hline Não & $91(61,5)$ \\
\hline \multicolumn{2}{|l|}{ Existência de outras comorbilidades médicas? $(n=154)$} \\
\hline Sim & $70(45,5)$ \\
\hline Não & $84(54,5)$ \\
\hline
\end{tabular}

perturbação de personalidade borderline, têm sido associadas a comportamentos impulsivos e autolesivos repetitivos. ${ }^{25}$ Esta característica reflete a dificuldade destes doentes na modulação e contenção das emoções. Os resultados deste estudo vão de encontro ao descrito, com uma associação positiva entre a perturbação de personalidade do cluster B (na qual se inclui a perturbação borderline) e os comportamentos autolesivos repetitivos ( $p=0,001)$.

Neste estudo, o uso de antidepressivos foi associado aos comportamentos suicidários de repetição, associação esta que tem sido abordada de forma controversa pela literatura. No nosso estudo, este resultado pode dever-se ao facto de um número muito elevado de doentes se encontrar sob medicação antidepressiva, não sendo possível localizar temporalmente o início da mesma. Será de considerar, contudo, a possibilidade de os antidepressivos, nomeadamente os inibidores seletivos da recaptação da serotonina (ISRS), contribuírem para o comportamento suicidário em determinados indivíduos, efeito adverso que deve ser considerado aquando da prescrição destes psicofármacos..$^{26-27}$

O estudo de Choi et al, em 2013, demonstrou existir maior risco de comportamentos autolesivos múltiplos em doentes com ideação suicida grave e relações interpessoais conflituosas. Demonstrou também relação significativa entre a história familiar de suicídio e estes comportamentos repetivos. ${ }^{28}$ Neste estudo não ficou demonstrado que os doentes com múltiplas tentativas de suicidas tinham mais conflitos que os res-

nero masculino, o consumo de substâncias de abuso teve uma associação significativa, independente do género, com o uso de métodos violentos. A maior letalidade no género masculino é também explicada por outras características do comportamento suicidário, para além do tipo de método. A utilização de mais do que um método e o menor número de pedidos de ajuda são mais frequentes no género masculino e estão associados a maior letalidade, ,920-23 apesar destes dados não se verificarem neste estudo.

\section{Fatores associados ao comportamento suicidário de repetição}

As perturbações de personalidade, particularmente a tantes. Do mesmo modo, também não se verificou associação positiva entre a história familiar de suicídio e os comportamentos autolesivos de repetição. No entanto, é importante referir que a variável «antecedentes familiares de suicídio» foi colhida em apenas $27 \%$ dos indivíduos.

\section{Comportamentos suicidários e cuidados de saúde primários}

Os comportamentos suicidários são frequentes nos cuidados de saúde primários (CSP). Um médico de família perde um paciente, vítima do suicídio, a cada quatro-sete anos. ${ }^{29-30}$ 
Em 2013, em Portugal, as perturbações depressivas afetavam cerca de $19,3 \%$ da população ao longo da vida. ${ }^{4} \mathrm{Um}$ estudo de 2014 refere que $10,4 \%$ dos doentes com depressão fará uma tentativa de suicídio em cinco anos. ${ }^{31}$ Cerca de dois terços dos doentes com comportamentos suicidários têm consulta com o seu médico de família nos meses que antecedem a tentativa de suicídio. ${ }^{32}$ Neste estudo, a maioria das pessoas (72\%) teve consulta nos CSP nos 12 meses prévios à tentativa de suicídio.

O médico de família, enquanto profissional de saúde, tem uma função privilegiada no conhecimento do indivíduo e sua família. Por esta razão, é fundamental que esteja atento a fatores preditores do comportamento suicidário para que possa agir atempadamente na sua prevenção. Conhecer as características da população que tenta o suicídio permite ao médico de família estar atento ao aparecimento das mesmas e atuar em conformidade.

Atendendo à relação entre depressão e comportamento suicidário, com sazonalidade potencial nos meses de inverno, bem como à associação entre o uso de antidepressivos e o comportamento suicidário, seria importante, por exemplo, uma maior monitorização dos pacientes com patologia depressiva nas fases iniciais da terapêutica antidepressiva e no período de inverno, com consultas mais regulares que permitissem uma avaliação psicopatológica adequada e o necessário suporte e/ou referenciação para os cuidados terciários (serviços hospitalares de psiquiatria). A valorização da presença de conflituosidade conjugal ou interpessoal nestes pacientes é também sustentada por este estudo e, nela, o médico de família poderá também ter um papel fundamental, atendendo à proximidade que assume em relação ao indivíduo e respetiva família.

Sendo o alcoolismo prevalente ao nível dos CSP e conhecida a associação deste com o uso de métodos violentos, seria recomendável a caracterização destes consumos, bem como a avaliação da ideação suicida ou autolesiva nos pacientes com risco suicidário, por exemplo, género masculino, com diagnóstico de doença psiquiátrica conhecido ou história familiar de comportamento suicidário. Aos indivíduos sinalizados poderiam ser fornecidas estratégias para redução dos consumos, envolvendo, oportunamente, o núcleo de suporte na comunidade e/ou familiar e, se necessário,

QUADRO VI. Estudo dos fatores relacionados com o comportamento repetidor e a utilização de métodos violentos

\section{Comportamento repetidor?}

\begin{tabular}{lccc}
\hline & Sim & & Não \\
\hline Género & & & 34 \\
Masculino & 10 & & 69 \\
Feminino & 40 & & \\
$P$ & & 0,095 & \\
\hline Vive sozinho? & & & 8 \\
Sim & 4 & & 95 \\
Não & 46 & & \\
$P$ & & 0,960 & \\
\hline Perturbação da personalidade? & & & 21 \\
Sim & 24 & & 82 \\
Não & 25 & & \\
$P$ & & 0,001 & \\
\hline Uso de antidepressivos? & & & 32 \\
Sim & 24 & & 68 \\
Não & 23 & & \\
$P$ & & 0,026 & \\
\hline Abuso de substancias?
\end{tabular}

\section{Abuso de substâncias?}

Sim

Não

9

15

34

80

$p$

Uso de método violento?

0,474

Sim

Não

\section{Género}

Masculino

Feminino

7

6 36

$p$

Vive sozinho?

Sim

Não 0 12

$P$ Perturbação da personalidade?

13

125

Sim

Não

$p$

Uso de antidepressivos?

Sim

Não

$p$

Abuso de substâncias

Sim

Não

$P$
3

10

43

93

0,755

$5 \quad 50$

$8 \quad 82$

1,000

5

8 18

105

0,045 
feita a referenciação para estruturas diferenciadas (serviços de psiquiatria, centros de respostas integradas).

\section{Limitações do estudo e pesquisa futura}

São limitações deste estudo o seu caráter retrospetivo e, portanto, dependente das informações registadas nos processos clínicos. Por outro lado, a reduzida amostra quando comparada com aquela que foi usada noutros estudos epidemiológicos é também um fator limitativo do caráter representativo dos resultados obtidos. Uma outra limitação do estudo prende-se com questões da logística do serviço de psiquiatria do HB, nomeadamente o facto de na UCS não estarem incluídos outros doentes com comportamentos suicidários, mas que mantiveram acompanhamento em consulta própria com o seu psiquiatra assistente (e.g., na consulta de psiquiatria geral ou consulta de espectro bipolar).

O desenho do estudo não permitiu a valorização da associação positiva entre os comportamentos suicidários de repetição e o uso de antidepressivos por não existirem dados que permitam discriminar se a utilização de medicação antidepressiva se iniciou antes ou depois do comportamento suicidário.

As limitações deste estudo influenciam naturalmente o impacto atribuído aos principais resultados obtidos, que estarão sobreestimados nalgumas situações, por exemplo, o estado civil casado e a situação profissional ativo ou a frequência do diagnóstico de perturbação da personalidade. Por outro lado, considera-se subestimada a frequência do consumo de substâncias, diagnósticos psiquiátricos como perturbação bipolar ou doenças do espectro da esquizofrenia e a história familiar de suicídio nos repetidores.

Por forma a aumentar a validade externa das conclusões obtidas neste trabalho, em estudos futuros seria importante a inclusão de um maior número de indivíduos, bem como a realização de estudos prospetivos que pudessem, por exemplo, incluir outras informações referentes à caracterização psicopatológica ou outros dados habitualmente não incluídos nos registos clínicos. Seria importante a comparação dos dados desta amostra com uma amostra de população com suicídio consumado, por forma a aferir a presença de diferenças em relação aos fatores de risco identificados.

A discussão de estratégias preventivas no que se refere ao comportamento suicidário de repetição e uso de método violento deverá ser futuramente estudada.

\section{REFERÊNCIAS BIBLIOGRÁFICAS}

1. Nock MK, Borges G, Bromet EJ, Cha CB, Kessler RC, Lee S. Suicide and suicidal behavior. Epidemiol Rev. 2008;30:133-54.

2. Saraiva CB, Peixoto B, Sampaio D. Suicídio e comportamentos autolesivos: dos conceitos à prática clínica. Lisboa: Lidel; 2014. ISBN 9789897520426

3. World Health Organization. Mental health: suicide data [homepage]. Geneva: WHO; 2016. Available from: http://www.who.int/mental_ health/prevention/suicide/suicideprevent/en/

4. Direção-Geral da Saúde. Portugal: saúde mental em números, 2013. Lisboa: DGS; 2013.

5. Schmidtke A, Bille-Brahe U, DeLeo D, Kerkhof A, Bjerke T, Crepet P, et al. Attempted suicide in Europe: rates, trends and sociodemographic characteristics of suicide attempters during the period 1989-1992 - Results of the WHO/EURO Multicentre Study on Parasuicide. Acta Psychiatr Scand. 1996;93(5):327-38.

6. Coimbra DG, Pereira e Silva AC, Sousa-Rodrigues CF, Barbosa FT, Siqueira Figueredo D, Araújo Santos JL, et al. Do suicide attempts occur more frequently in the spring too? A systematic review and rhythmic analysis. J Affect Disord. 2016;196:125-37.

7. Sansone RA, Sansone LA. Sunshine, serotonin, and skin: a partial explanation for seasonal patterns in psychopathology? Innov Clin Neurosci. 2013;10(7-8):20-4.

8. Ajdacic-Gross V,Weiss MG, Ring M, Hepp U, Bopp M, Gutzwiller F, et al. Methods of suicide: international suicide patterns derived from the WHO mortality database. Bull World Health Organ. 2008;86(9):726-32.

9. Cibis A, Mergl R, Bramesfeld A, Althaus D, Niklewski G, Schmidtke A, et al. Preference of lethal methods is not the only cause for higher suicide rates in males. J Affect Disord. 2012;136(1-2):9-16.

10. Värnik A, Kölves K, van der Feltz-Cornelis CM, Marusic A, Oskarsson H, Palmer $A$, et al. Suicide methods in Europe: a gender specific analysis of countries participating in the "European Alliance Against Depression". J Epidemiol Community Health. 2008;62(6):545-51.

11. Värnik $A$, Sisask $M$, Värnik $P, W u$ J, Kõlves $K$, Arensman $E$, et al. Drug suicide: a sex-equal cause of death in 16 European countries. BMC Public Health. 2011;11(1):61.

12. Hawton K. Sex and suicide: gender differences in suicidal behaviour. Br J Psychiatry. 2000;177:484-5.

13. Bertolote JM, Fleischmann A, De Leo D, Wasserman D. Suicide and mental disorders: do we know enough? Br J Psychiatry. 2003;183:382-3.

14. Younes N, Melchior M, Turbelin C, Blanchon T, Hanslik T, Chee CC. Attempted and completed suicide in primary care: not what we expected? J Affect Disord. 2015;170:150-4.

15. Craveiro A, Veiga FA, Vicente C, Tavares A, Vinhas C, Santos Z, et al. Epidemiologia do para-suicídio no concelho de Coimbra (anos de 1994-19951996-1997). Psiq Clin. 1998;19:283-8.

16. Welch SS. A review of the literature on the epidemiology of parasuicide in the general population. Psychiatr Serv. 2001;52(3):368-75.

17. Poma SZ, Magno N, Belletti S, Toniolo E. Parasuicide in Rovigo (North of Italy) during the period 2000-2005. J Prev Med Hyg. 2007;48(3):79-82.

18. Lester D. Seasonal variation in suicidal deaths by each method. Psychol Rep. 1985;56(2):650.

19. Stack S, Wasserman I. Race and method of suicide: culture and opportunity. Arch Suicide Res. 2005;9(1):57-68.

20. Racette S, Sauvageau A. Planned and unplanned complex suicides: a 5-year 
retrospective study. J Forensic Sci. 2007;52(2):449-52.

21. Töro K, Pollak S. Complex suicide versus complicated suicide. Forensic Sci Int. 2009;184(1-3):6-9.

22. Möller-Leimkühler AM. The gender gap in suicide and premature death or: why are men so vulnerable? Eur Arch Psychiatry Clin Neurosci. 2003;253(1):1-8

23. Houle J, Mishara BL, Chagnon F. An empirical test of a mediation model of the impact of the traditional male gender role on suicidal behavior in men. J Affect Disord. 2008;107(1-3):37-43.

24. Boenisch S, Bramesfeld A, Mergl R, Havers I, Althaus D, Lehfeld H, et al. The role of alcohol use disorder and alcohol consumption in suicide attempts: a secondary analysis of 1921 suicide attempts. Eur Psychiatry. 2010;25(7):414-20.

25. American Psychiatric Association. Practice guideline for treatment of patients with borderline personality disorder. Washington, DC: APA; 2010.

26. Stone M, Laughren T, Jones ML, Levenson M, Holland PC, Hughes A, et al. Risk of suicidality in clinical trials of antidepressants in adults: analysis of proprietary data submitted to US Food and Drug Administration. BMJ. 2009;339:b2880

27. Gibbons RD, Brown CH, Hur K, Davis J, Mann JJ. Suicidal thoughts and behavior with antidepressant treatment: reanalysis of the randomized placebo-controlled studies of fluoxetine and venlafaxine. Arch Gen Psychiatry. 2012;69(6):580-7.
28. Choi KH, Wang SM, Yeon B, Suh SY, Oh Y, Lee HK, et al. Risk and protective factors predicting multiple suicide attempts. Psychiatry Res. 2013;210(3):957-61.

29. Gunnell D, Bennewith O, Peters TJ, Stocks N, Sharp DJ. Do patients who selfharm consult their general practitioner soon after hospital discharge? A cohort study. Soc Psychiatry Psychiatr Epidemiol. 2002;37(12):599-602.

30. Marquet RL, Bartelds AI, Kerkhof AJ, Schellevis FG, Van der Zee J. The epidemiology of suicide and attempted suicide in Dutch general practice 19832003. BMC Fam Pract. 2005;6:45.

31. Riihimäki K, Vuorilehto M, Melartin T, Haukka J, Isometsä E. Incidence and predictors of suicide attempts among primary-care patients with depressive disorders: a 5-year prospective study. Psychol Med. 2014;44(2):291-302.

32. Olfson M, Weissman MM, Leon AC, Sheehan DV, Farber L. Suicidal ideation in primary care. J Gen Intern Med. 1996;11(8):447-53.

\section{CONFLITO DE INTERESSES}

Os autores declaram não ter conflitos de interesses.

\section{ENDEREÇO PARA CORRESPONDÊNCIA}

E-mail: joana.castanheira@gmail.com

Recebido em 23-07-2016

Aceite para publicação em 29-08-2017

\section{ABSTRACT}

\section{SUICIDAL BEHAVIOUR: CHARACTERISATION AND DISCUSSION OF VULNERABILITY FACTORS}

Objectives: To characterise the group of patients that had the first medical appointment in the Suicidal Behaviour Unit (SCU) of Braga Hospital (HB) psychiatric service during the years 2014-2015 and identify features associated with the higher risk of repeating behaviour and use of violent methods.

Type of study: Observational, cross-sectional, descriptive and analytical.

Location: Braga Hospital.

Population: Patients who had the first appointment in HB psychiatry service UCS in the period between 01/01/2014 and 31/12/2015. Methods: Collection of routine data using de clinical file. Collection of sociodemographic, psychosocial, and clinical circumstances of suicidal behaviour. SPSS software, v. 21, was used to analyse and process the data. Univariate and bivariate descriptive analysis were performed. Then a binary logistic regression model was created to identify possible risk factors associated with repeated behaviours and more violent methods.

Results: The study included 154 individuals, mostly women (70.8\%) with average age of 39.7 years. Most of them were married (51.9\%). A higher number of suicide attempts were observed during months whose days have less sun. Drug intake was the most frequently used method (68.2\%). The most violent and lethal methods were more used by men $(p=0.036)$. The use of substances was associated with the practice of more violent suicidal attempts $(p=0.045)$. Personality disorder $(p=0.001)$ and treatment with antidepressants $(p=0.028)$ were risk factors for suicidal behaviour repetition. In this study, most of individuals $(72 \%)$ had an appointment in primary care, in the 12 months prior to the suicidal attempt.

Conclusions: In this study the most frequent diagnosis was depression. This study allowed to identify personality disorders and the use of antidepressant medication as risk factors associated with repetitive suicidal behaviour. Our results point male gender and substance abuse as risk factors to the use of more violent and lethal methods. This knowledge is essential for the prevention of mortality associated with suicidal behaviour, especially among family doctors. However, more studies are needed to strengthen the influence of the risk factors in the population.

Keywords: Suicide; Vulnerability study. 